\title{
Functional hemispherectomy for patients with adult-onset Rasmussen's encephalitis
}

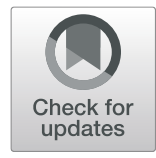

\author{
Hong Xue ${ }^{1,2+}$, Cuiping $\mathrm{Xu}^{1+}$, Xiaoxia Zhou', Duanyu $\mathrm{Ni}^{1}$, Xueyuan Wang ${ }^{1}$ and Tao Yu ${ }^{1 *}$ (1)
}

\begin{abstract}
Background: Surgical treatment for patients with adult-onset Rasmussen's encephalitis (A-RE) is rarely reported. We investigated the clinical and surgical features of two patients with A-RE who underwent functional

hemispherectomy.

Case presentation: The data of clinical manifestations, neuroimaging, surgical treatment and surgical outcomes of two patients with A-RE was reviewed. The two patients initially presented with recurrent partial seizures or secondly generalized tonic clonic seizures. Gradually, the patients showed unilateral limb paralysis as well as chronic focal epileptic status. Both patients underwent functional hemispherectomy and achieved seizure freedom in the followup. The contralateral neurological deficits improved gradually after rehabilitation and were acceptable for the selfcare of daily living. The living quality improved prominently after surgery.

Conclusions: Despite the risk of hemiplegia, functional hemispherectomy may be a choice for patients with A-RE for favorable seizure control and improved quality of life in selected patients.
\end{abstract}

Keywords: Rasmussen encephalitis, Adult, Epilepsy, Hemispherectomy

\section{Background}

Rasmussen's encephalitis (RE), which was first reported in 1958 by professor Rasmussen, is a rare immune-mediated condition that is characterized by drug-resistant focal epilepsy, progressive neurological, and cognitive deficits associated with unilateral hemispheric atrophy $[1,2]$. Diagnosis criteria consist of clinical, electroencephalogram (EEG) and magnetic resonance imaging (MRI) features. However, Early diagnosis is particular challenge. RE is generally found in children with an onset age peak of approximately 6 years. Anti-epilepsy drugs are limited to secondarily generalized seizures and complex partial seizures, whereas epilepsia partialis continua is typically totally refractory [2]. Surgery plays a major role in the treatment of childhood RE and aims to control seizures and interrupt the evolution of the structural encephalitic process.

\footnotetext{
* Correspondence: yutaoly@sina.com

${ }^{\dagger}$ Hong Xue and Cuiping Xu contributed equally to this work.

${ }^{1}$ Beijing Institute of Functional Neurosurgery, Xuanwu Hospital, Capital

Medical University, No. 45 Changchun Street, Beijing 100053, China

Full list of author information is available at the end of the article
}

Because most of the lesions are limited to one hemisphere, functional hemispherectomy and hemispherotomy are the final effective treatments for controlling the seizures in these patients, although there are some neurological sequels [3]. For most children with RE, these disconnective techniques constitute an extremely effective method to terminate seizures. Moreover, the contralateral disabled limbs may obtain good functional recruitment with effective compensation and can obviously improve the patient's quality of life.

However, RE in adults is rare. There is little medical experience with the progression of $\mathrm{RE}$ initiation in adults. Whether surgical resection of damaged hemispheres is beneficial, as well as the degree of postoperative functional compensation, is unknown. The disconnective approach may not be feasible in adults with preserved neurological functions because of the heavy neurological morbidity [4].

We report two special patients with adult-onset RE (A-RE) who received functional hemispherectomy at Xuanwu hospital. Surgical treatment for patients with A-

(c) The Author(s). 2021 Open Access This article is licensed under a Creative Commons Attribution 4.0 International License, which permits use, sharing, adaptation, distribution and reproduction in any medium or format, as long as you give appropriate credit to the original author(s) and the source, provide a link to the Creative Commons licence, and indicate if changes were made. The images or other third party material in this article are included in the article's Creative Commons licence, unless indicated otherwise in a credit line to the material. If material is not included in the article's Creative Commons licence and your intended use is not permitted by statutory regulation or exceeds the permitted use, you will need to obtain permission directly from the copyright holder. To view a copy of this licence, visit http://creativecommons.org/licenses/by/4.0/. 
$\mathrm{RE}$ is rarely reported. We discuss the clinical features, surgical treatment, and surgical outcome of patients with A-RE who underwent epilepsy surgery. The use of sample from the patients was approved by the Institutional Ethics Committee of Xuan Wu hospital Capital Medical University and informed consents have been obtained from patients prior to analysis.

\section{Case presentation}

Case 1

This male patient was 33 years old and had complained of paroxysmal convulsions for 5 months. He was admitted to the hospital in 2017. Five months prior to admission, the patient had a sudden evolving tonic-clonic convulsion without obvious inducement from the left limb to the right limb with the loss of consciousness, which lasted for approximately $1 \mathrm{~min}$. The frequency of the convulsion increased gradually to one to 20 times per day in the subsequent months. A video EEG examination was performed at the local hospital, and $0.5 \mathrm{~g}$ sodium valproate tablets were prescribed twice daily. However, the effect was not obvious. After an additional prescription of levetiracetam, the seizures were slightly reduced to 4 times per day. After admission to our hospital, nervous system examination showed that the muscle force decreased to grade III in left upper limb and grade IV in the left lower extremity with increased muscular tension. The patient had tendon hyperreflexia in the left limbs. Somatosensory evoked potential showed that the left median nerve stimulation did not lead to action potential, whereas right median nerve stimulation elicited action potential. Stimulation of the left central cortex induced right limb movement, and stimulation of the right central cortex failed to lead to movement of the left limbs. MRI showed obvious progressive atrophy of the right hemisphere (Fig. 1). In conclusion, RE was clinically diagnosed for this patient. Based on the results above, right functional hemispherectomy was performed. After surgery, the patient exhibited weakness of the left upper and lower limb. After rehabilitation for 3 months, the patient walked independently with weakness of the left hand and foot. Antiepileptic medication remained the same as before surgery. The patient has been seizure-free since surgery and had good self-care ability after 6 months of rehabilitation.

\section{Case 2}

This male patient was 29 years old and complained of paroxysmal convulsions for more than 3 years. He was admitted to the hospital in 2012. Three years prior to admission, the patient had a sudden tonic-clonic convulsion without obvious inducement with the loss of consciousness and urinary incontinence. After a few minutes of remission, the seizure recurred again and again until sedative administration in the hospital $1 \mathrm{~h}$ later. The local hospital prescribed many antiepileptic drugs to control the seizures in the subsequent days. Dexamethasone and oral hormones were also prescribed, but there was no optimal effect. Furthermore, left limb mild hemiparesis occurred after the continuing seizures. The patient had one to six seizures per day in the subsequent months with the regular use of antiepileptic drugs. The left hemiparesis developed gradually, and he could not walk independently for more than 1 year. MRI showed abnormal signals in the right frontoparietal lobe in an early stage. Then, right hemispheric atrophy occurred (Fig. 2). EEG showed continuous slow wave in the right hemisphere. Magnetoencephalography showed interictal dipoles concentrated in the right central-parietal region and scattered in the right middle-posterior temporal region. Based on these results, a right functional hemispherectomy was performed in our hospital. The patient was immediately seizure free after surgery. The weakness of the left upper and lower limb was aggravated in the early postoperative stage and improved gradually after rehabilitation in the following 12 months. The antiepileptic medications were decreased gradually in the following 7 years, and he could walk independently 2 years after surgery.

\section{Discussion}

RE also known as Rasmussen syndrome, is an acquired, progressive, chronic inflammatory brain disease that develops in childhood, and it is characterized by refractory epilepsy, progressive hemiplegia, and mental decline in one hemisphere of the brain [1]. However, RE with adult onset is rare, and it was reported in only a few publications [5-7]. A-RE is reported with an atypical presentation and a slower and milder course in which motor and speech function are preserved [8]. Compared with childhood RE patients, the A-RE patients had atypical electro-clinical features and less severe progression of the structural brain atrophy. It needed to be differentiated from hemiconvulsion-hemiplegia-epilepsy syndrome, focal cortical dysplasia and tuberous sclerosis. Therefore, a later correct diagnosis or misdiagnosis may occur. This milder progression of the structural brain atrophy is thought to be related to the selective susceptibility of specific brain structures to immunological or inflammatory processes $[9,10]$.

Both of the patients in our report had typical refractory epilepsy and progressive hemiplegia. The two patients progressed to obvious hemiplegia in a few months. The MRI showed a prominent progressive hemispheric atrophy in patient 1 and relative milder alterations in patient 2 .

Although some therapeutic options, such as new antiepileptic drugs or immunomodulatory agents, are now 
a

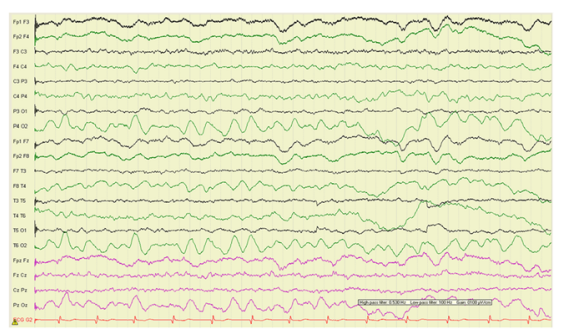

c

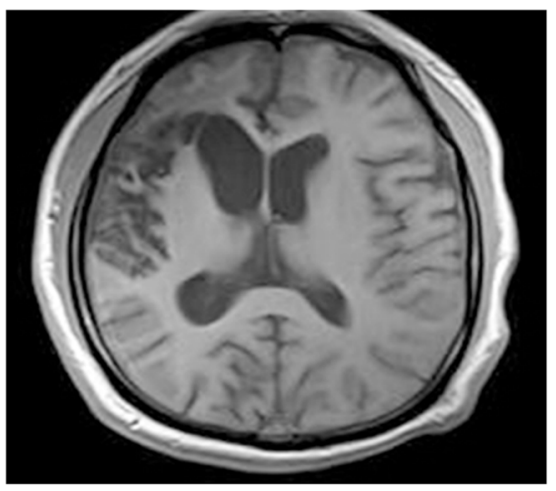

e

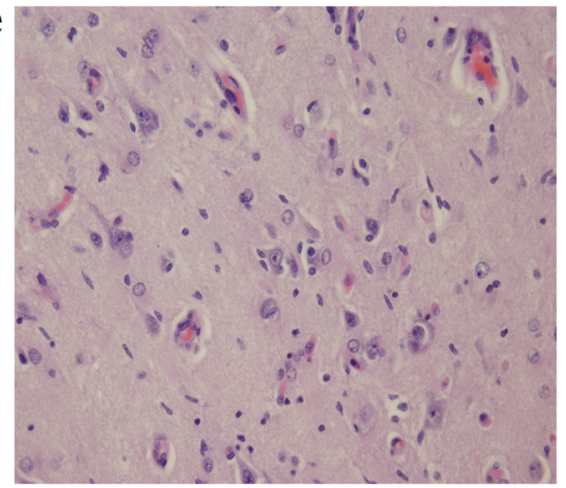

b

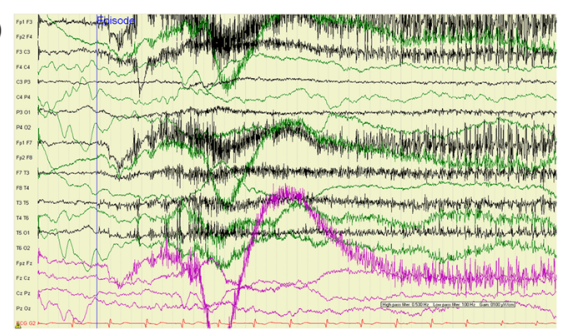

d
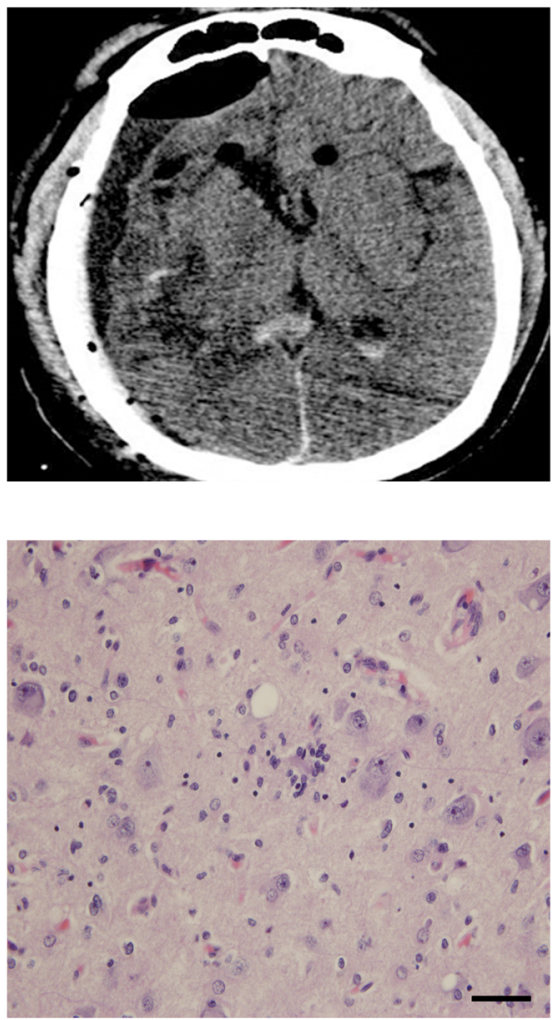

Fig. 1 Clinical data of case 1. a Interictal EEG showed slow wave on the right temporal and parietal regions. b Ictal EEG showed the electromyographic artifact and reduced electrical activity on the right hemisphere. c Axial MRI showed atrophy of the right hemisphere. d Postoperative CT. e Pathological images showed diffuse infiltration of microglia lymphocytes and neuronophagia phenomenon. Scale bar: $50 \mu \mathrm{m}$

available for RE patients, seizure freedom is never completely achieved, and the epilepsia partialis continua is generally "super-refractory" [11, 12]. Therefore, surgery remains a choice of treatment. Functional hemispherectomy or hemispherotomy is the most effective therapy to achieve long-term seizure freedom for $62.5-85 \%$ of patients, but some neurological complications occur [2]. However, the experience of surgery for patients with A$\mathrm{RE}$ is limited. Some authors preferred focal resection, but others suggested hemispherectomy [6]. Limited cortical resection achieved sustained seizure freedom [4]. One possible explanation of a favourable surgical outcome following limited cortical resection is that the surgical procedure produced seizure network disruption. Unfortunately, the favourable surgical outcome in that study was not paralleled by the inhibition of neurological progression in one of the two cases. Selective cortical resection may not change the progressive nature of this disease. Limited cortical resections are only transiently effective [9]. Therefore, functional hemispherotomy is suggested, rather than a conservative surgical approach, to obtain favourable surgical outcome.

The lesions in these two patients located in the nondominant hemisphere, which was relatively simple for surgical selection. However, if the lesion is located in the dominant hemisphere of the patient, the extent of impaired speech and contralateral compensation needs to be adequately assessed. As we know, the compensatory ability of language function in adults is significantly weaker than that in children. The common methods we used to assess the extent of language function are functional magnetic resonance imaging (fMRI) of language 
$\mathbf{a}$

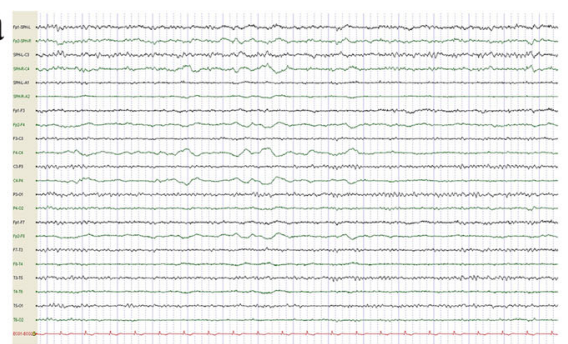

c

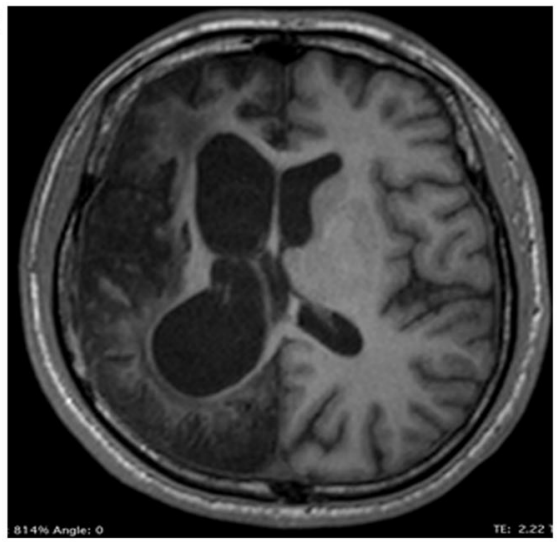

e

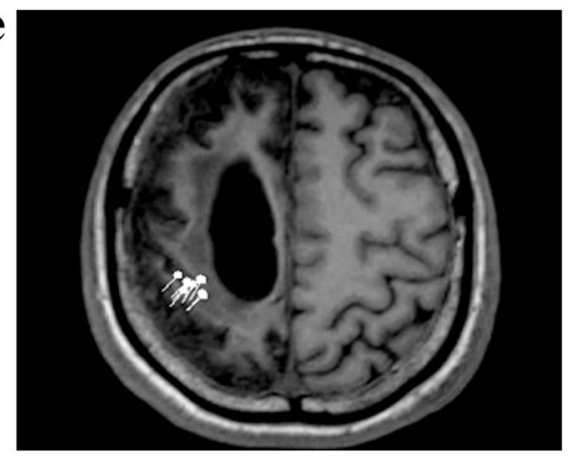

$\mathbf{f}$

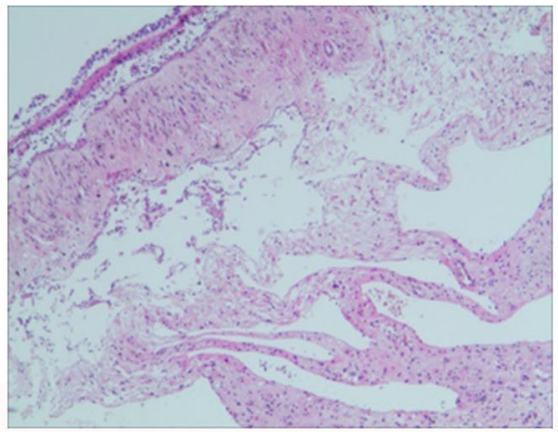

b

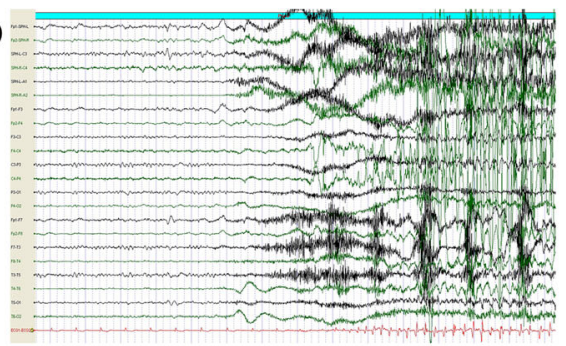

d
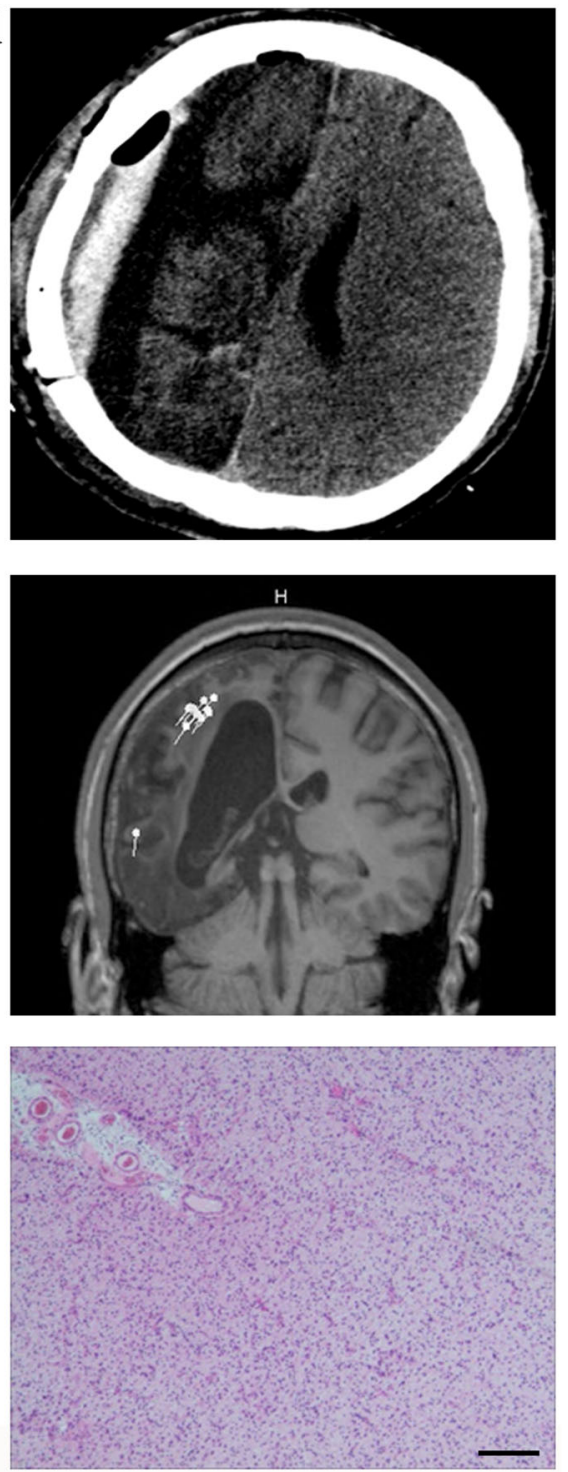

Fig. 2 Clinical data of case 2. a Interictal EEG showed slow wave on the right frontal, temporal and parietal regions. b Ictal EEG showed slow wave on the right frontal and temporal regions. c Axial MRI showed atrophy of the right hemisphere. $\mathbf{d}$ Post-operative CT. e MEG showed interictal dipoles concentrated in the right central-parietal region and scattered in the right middle-posterior temporal region. $\mathbf{f}$ Pathological result showed cicatricial gyrus formation. Scale bars: $100 \mu \mathrm{m}$

tasks and transcranial magnetic stimulation (TMS). There is still an opportunity to perform hemispherectomy if the patient develops significant speech impairment with evidence of contralateral compensation on either fMRI or TMS. Otherwise, hemispherectomy may not be recommended. As for the selection of hemispherical operations, the main consideration is the compensatory ability of the contralateral hemisphere, including 
the volumetric compensation. As we know, the compensatory ability is relatively weak in adults. Therefore, we didn't applied anatomical hemispherectomy avoiding large intracranial cavity. Both functional hemispherectomy and hemispherical disconnection are suitable. Another consideration for the choice of functional hemisphrectomy in our two patients was the diagnosis. Because the A-RE was rare, the pathological examination was help for confirming the diagnosis of RE. Therefore, we performed functional hemispherectomy and removed some part of the damaged brain tissue in the intraoperative exposure.

Both patients in our report underwent functional hemispherectomy and had sustained seizure freedom, but hemiplegia was aggravated instantly after surgery. Fortunately, the hemiplegia was also gradually alleviated after rehabilitation. Although the hemiplegia was obvious, both patients could walk independently and achieved a satisfying postoperative quality of life.

\section{Conclusion}

Despite the risk of neurological impairments, especially hemiplegia, functional hemispherectomy may be a choice for patients with A-RE for a favourable surgical outcome and improved quality of life in selected patients.

\section{Abbreviations}

RE: Rasmussen encephalitis; A-RE: Adult-onset Rasmussen encephalitis; EEG: Electroencephalogram; MRI: Magnetic resonance imaging;

TMS: Transcranial magnetic stimulation

\section{Acknowledgements}

We would like to thank professor Yueshan Piao for the pathological data. We also thank the patient and her family for coordination and all medical staff in the department of functional neurosurgery of Xuanwu Hospital Capital Medical University for providing medical care for the patient.

\section{Disclosure}

We confirm that we have read the Journal's position on issues involved in ethical publication and affirm that this report is consistent with those guidelines.

\section{Authors' contributions}

Doctor Hong Xue and Cuiping Xu contributed equally to this work and they are co-first authors. Professor Tao Yu is the corresponding author. Hong Xue collected the clinical data and wrote the original draft perparation. Cuiping Xu wrote and revised the paper. Xiaoxia Zhou is contributed to modification and validation. Duanyu Ni is contributed to surgeon supervision. Xueyuan Wang is contributed to surgeon investigation. Tao Yu is contributed to writing, reviewing and editing. The authors read and approved the final manuscript.

\section{Funding}

This study was supported by National Natural Science Foundation of China (Grant No. 81771395).

Availability of data and materials

Please contact authors for data requests.

\section{Declarations}

Ethics approval and consent to participate

The use of sample from the patients was approved by the Institutional Ethics Committee of Xuan Wu hospital Capital Medical University and informed consents have been obtained from patients prior to analysis.

\section{Consent for publication}

All authors agree to publish the study. The informed consent about publication was obtained from the patient.

\section{Competing interests}

The authors declared no commercial or financial conflict of interest.

\section{Author details}

'Beijing Institute of Functional Neurosurgery, Xuanwu Hospital, Capital Medical University, No. 45 Changchun Street, Beijing 100053, China.

${ }^{2}$ Department of Neurology, The second Clinical Medical College of Guizhou University of traditional Chinese Medicine, Guiyang 550001, Guizhou, China.

Received: 18 November 2020 Accepted: 13 May 2021

Published online: 28 May 2021

References

1. Varadkar S, Bien CG, Kruse CA, Jensen FE, Bauer J, Pardo CA, et al. Rasmussen's encephalitis: clinical features, pathobiology, and treatment advances. Lancet Neurol. 2014;13(2):195-205. https://doi.org/10.1016/S14 74-4422(13)70260-6.

2. Bien CG, Granata T, Antozzi C, Cross JH, Dulac O, Kurthen M, et al. Pathogenesis, diagnosis and treatment of Rasmussen encephalitis: a European consensus statement. Brain. 2005;128(3):454-71. https://doi.org/1 0.1093/brain/awh415.

3. Schramm J, Kral T, Clusmann H. Transsylvian keyhole functional hemispherectomy. Neurosurgery. 2001;49:891-900 discussion 900-891.

4. Villani F, Didato G, Deleo F, Tringali G, Garbelli R, Granata T, et al. Long-term outcome after limited cortical resections in two cases of adult-onset Rasmussen encephalitis. Epilepsia. 2014;55(5):e38-43. https://doi.org/1 0.1111/epi.12596.

5. Abd-El-Barr MM, Wu B, Rahman M, Yachnis AT, Roper SN, Eisenschenk S. Atypical Rasmussen's encephalitis treated with temporal lobectomy. J Clin Neurosci. 2011;18(2):287-90. https://doi.org/10.1016/j.jocn.2010.05.011.

6. Cheong JY, Wong C, Bleasel A, Varikatt W, Ng T, Dexter MA. Late onset Rasmussen's encephalitis with triple pathology. J Clin Neurosci. 2009;16(12): 1677-81. https://doi.org/10.1016/j.jocn.2009.02.042.

7. Hennessy MJ, Koutroumanidis M, Dean A, Jarosz J, Elwes RD, Binnie CD, et al. Chronic encephalitis and temporal lobe epilepsy: a variant of Rasmussen's syndrome? Neurology. 2001;56(5):678-81. https://doi.org/1 0.1212 WNL.56.5.678

8. Villani F, Pincherle A, Antozzi C, Chiapparini L, Granata T, Michelucci R, et al. Adult-onset Rasmussen's encephalitis: anatomical-electrographic-clinical features of 7 Italian cases. Epilepsia. 2006;47(Suppl 5):41-6. https://doi.org/1 0.1111/j.1528-1167.2006.00876.x.

9. Korn-Lubetzki I, Bien CG, Bauer J, Gomori M, Wiendl H, Trajo L, et al. Rasmussen encephalitis with active inflammation and delayed seizures onset. Neurology. 2004;62(6):984-6. https://doi.org/10.1212/01.WNL.000011 5393.67403.53.

10. Takahashi Y, Mori H, Mishina M, Watanabe M, Kondo N, Shimomura J, et al. Autoantibodies and cell-mediated autoimmunity to NMDA-type GluRepsilon2 in patients with Rasmussen's encephalitis and chronic progressive epilepsia partialis continua. Epilepsia. 2005;46(Suppl 5):152-8. https://doi.org/10.1111/j.1528-1167.2005.01024.x.

11. Granata T, Fusco L, Gobbi G, Freri E, Ragona F, Broggi G, et al. Experience with immunomodulatory treatments in Rasmussen's encephalitis. Neurology. 2003;61(12):1807-10. https://doi.org/10.1212/01.WNL. 0000099074.04539.E0.

12. Villani F, Spreafico R, Farina L, Giovagnoli AR, Bernasconi P, Granata T, et al. Positive response to immunomodulatory therapy in an adult patient with Rasmussen's encephalitis. Neurology. 2001;56(2):248-50. https://doi.org/1 0.1212/WNL.56.2.248. 